\title{
磷烯光催化分解水研究进展
}

\author{
郑 云 $^{1,2}$, 陈亦琳 ${ }^{1}$, 高碧芬 ${ }^{1}$, 林碧洲 ${ }^{1}$
}

(1. 华侨大学 材料科学与工程学院, 厦门 361021; 2. 福州大学 化学学院, 能源与环境光催化国家重点实验室, 福 州 350116)

摘 要: 半导体光催化分解水被认为是解决全球能源短缺和环境污染问题的潜在途径之一。近年来, 磷烯(BP)由于 具有带隙可调、空穴迁移率高、吸收光谱宽等特性而在光催化分解水方面得到了广泛关注。本文综述了国内外近 年来在磷烯光催化分解水领域所取得的重要研究进展, 总结了磷烯基光催化剂的合成方法、表面修饰和异质结构构 建等改性策略，阐述了磷烯基光催化剂的构-效关系和电荷转移机制，并展望了磷烯基光催化剂所面临的机遇和挑 战, 揭示了磷烯基材料在太阳能利用和转化方面的重要应用潜力。

关 键 词: 磷烯; 光催化; 分解水; 二维材料; 综述

中图分类号: O643 文献标识码: A

\section{Progress on Phosphorene for Photocatalytic Water Splitting}

\author{
ZHENG Yun $^{1,2}$, CHEN Yilin ${ }^{1}$, GAO Bifen ${ }^{1}$, LIN Bizhou $^{1}$
}

(1. College of Materials Science and Engineering, Huaqiao University, Xiamen 361021, China; 2. State Key Laboratory of Photocatalysis on Energy and Environment, College of Chemistry, Fuzhou University, Fuzhou 350116, China)

\begin{abstract}
Semiconductor photocatalytic water splitting has been considered as a potential strategy to overcome global energy shortage and environmental pollution. In recent years, phosphorene (BP) attracted great attention in photocatalytic water splitting due to its adjustable band gap, high hole mobility and wide absorption spectrum. This review summarizes the recent significant advances on designing high-performance BP-based photocatalysts for water splitting. The synthetic methods and modification strategies (e.g., surface modification and heterostructure design) of BP-based photocatalysts are described. Furthermore, in order to elucidate the structure-activity relationship of BP-based photocatalysts, the charge transfer mechanism is illustrated. Finally, the ongoing challenges and opportunities for the future development of BP-based photocatalysts in the exciting research area are highlighted.
\end{abstract}

Key words: phosphorene; photocatalysis; water splitting; two-dimensional materials; review

利用光催化分解水来获取清洁的氢能源，是解 决能源危机和环境问题的理想途径之一, 受到各国 科学家的广泛关注 ${ }^{[1]}$ 。近四十年来, 金属氧化物、硫
化物、氮氧化物、磷酸盐、卤氧铋、聚合物半导体 及其复合物等多种光催化剂已被用于光催化分解 水 $^{[2-10]}$, 然而, 还没有一种单独的半导体可以同时

收稿日期: 2019-06-24; 收到修改稿日期：2019-08-23

基金项目: 国家自然科学基金(21902051); 福建省自然科学基金(2019J05090, 2017J01014); 福建省石墨烯复合材料研究中 心(2017H2001); 华侨大学科研基金(605-50Y17060); 福州大学能源与环境光催化国家重点实验室开放课题 (SKLPEE-201803)

National Natural Science Foundation of China (21902051); Natural Science Foundation of Fujian Province (2019J05090, 2017J01014); Graphene Power and Composite Research Center of Fujian Province (2017H2001); Scientific Research Funds of Huaqiao University (605-50Y17060); The Open Project Program of the State Key Laboratory of Photocatalysis on Energy and Environment of Fuzhou University (SKLPEE-201803)

作者简介: 郑云(1990-), 女, 博士，讲师. E-mail: zheng-yun@hqu.edu.cn ZHENG Yun(1990-), female, PhD, lecturer. E-mail: zheng-yun@hqu.edu.cn 
满足高效光催化剂的所有要求。宽带隙半导体具有 较好的稳定性但光吸收范围有限, 窄带隙的半导体 (例如 $\mathrm{CdS}$ ) 能吸收范围更宽的太阳光, 但在光催化 过程中容易发生光腐蚀。此外, 只有极少数窄带隙 半导体可以吸收占太阳光谱能量 $40 \%$ 以上的近红外 (NIR)光。因此, 开发具有高量子效率和宽光谱响应 的光催化剂是当前光催化领域的研究重点之一。

磷烯(BP) 是一种通过机械剥离法从黑磷中分离 出来的单元素二维材料 ${ }^{[11]}$ 。磷烯因其具有二维层状结 构、可调的带隙宽度、高空穴迁移率、能吸收紫外到 近红外光的特性, 近年来在光催化领域引起了广泛 关注 ${ }^{[12]}$ 。但是由于磷烯的光生载流子分离效率低, 其光催化分解水效率还较低。通过对磷烯进行表面 修饰、异质结构构筑等方法能够提高光生载流子的 分离效率, 从而提升光催化分解水性能。

本文总结了磷烯基光催化剂在可见光和近红外 光下分解水的研究进展, 阐述了磷烯基光催化剂的 合成策略、表面修饰和异质结构设计等改性方法, 并对该领域未来的研究方向进行了展望(图 1)。

\section{1 磷烯光催化裂解水制氢}

\section{1 磷烯光催化裂解水的理论研究}

科学家们通过密度泛函理论(DFT)计算从理论 上预测了 BP 作为光催化剂应用于分解水制取氢气 的可能性。如图 2 所示, 无论在一般条件下还是在 拉伸应变条件下, 在 $\mathrm{pH}=8.0$ 的溶液中磷烯的导带底 $(\mathrm{CBM})$ 的位置比 $\mathrm{H}^{+} / \mathrm{H}_{2}$ 电位更负, 而价带顶(VBM) 的位置比 $\mathrm{O}_{2} / \mathrm{H}_{2} \mathrm{O}$ 电位更正, 能较好地满足光催化分 解水的要求 ${ }^{[13]}$ 。磷烯在沿 $a$ 轴 $7 \%$ 拉伸应变下的带

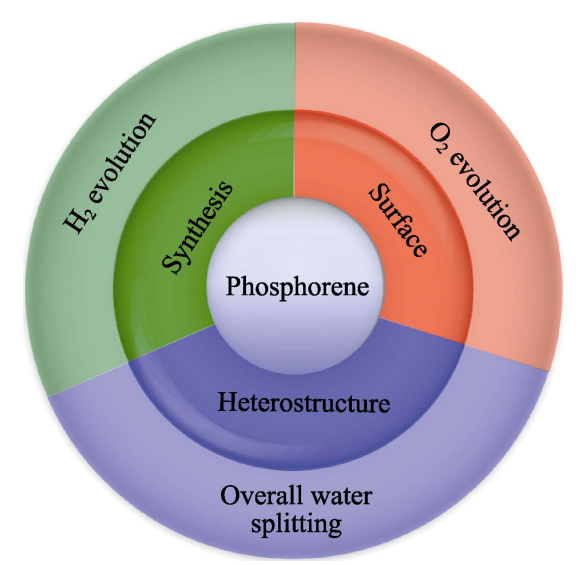

图 1 磷烯的合成、表面修饰、异质结构设计及光催化分解 水应用

Fig. 1 Synthesis, surface modification and heterostructure design of phosphorene-based photocatalysts for half-reactions and overall reactions of water splitting

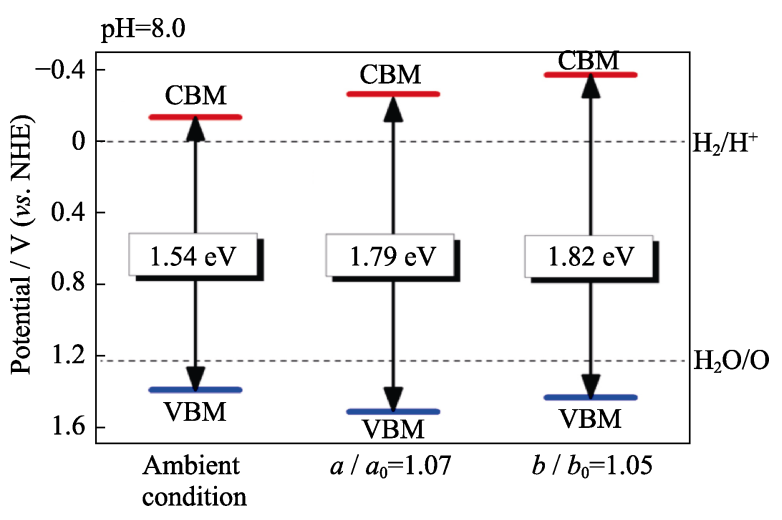

图 2 在 $\mathrm{pH}=8.0$ 的一般条件下沿 $a$ 轴 7\%拉伸应变, 沿 $b$ 轴 $5 \%$ 拉伸应变时磷烯的能带图 ${ }^{[13]}$

Fig. 2 Band edge alignments of phosphorene at ambient condition, under $7 \%$ tensile strain along $a$ axis and $5 \%$ tensile strain along $b$ axis when $\mathrm{pH}=8.0^{[13]}$

隙为 $1.79 \mathrm{eV}$, 在沿 $b$ 轴 $5 \%$ 拉伸应变下的带隙为 $1.82 \mathrm{eV}$ 。这些带隙位于可见光波长范围内, 能够有 效地捕获可见光 ${ }^{[13]}$ 。由于磷烯在一定的 $\mathrm{pH}$ 条件下 具有合适的带隙和能带位置, 可作为一种高效的可 见光驱动水分解光催化剂。这些理论计算研究预测 了磷烯在光催化分解水方面的应用潜力。

\section{2 二维磷烯光催化剂的合成及表面修饰}

目前, 二维磷烯纳米片光催化剂的合成方法主 要可分为自上而下法和自下而上法。通过机械球磨、 液相超声剥离等自上而下的方法可从体相黑磷中剥 离出少层磷烯纳米片 ${ }^{[14]}$ 。磷烯纳米片可作为一种可 见光响应的光催化剂用于光催化产氢 ${ }^{[15]}$ 。如图 3 所 示, 通过机械球磨方法在 $\mathrm{LiOH}$ 添加剂的作用下实 现了对体相黑磷的剥离。在没有任何助催化剂存在 的条件下, 剥离后的磷烯 (BP-BM)纳米片在可见光 下的产氢速率为 $512 \mu \mathrm{mol} \cdot \mathrm{h}^{-1} \cdot \mathrm{g}^{-1}$, 约是体相黑磷 (Bulk BP)的 18 倍。BP-BM 光催化产氢速率的提高 主要归因于球磨剥离后黑磷带隙的增大, 使导带与 价带的位置更有利于光生载流子的快速分离, 从而 促进质子的还原产生氢气。

通过水热、溶剂热等自下而上的方法可实现少 层磷烯纳米片的可控合成。以白磷 $\left(\mathrm{P}_{4}\right)$ 为原料, 乙二 胺 (EN) 为溶剂, 通过溶剂热法制备了克数量级的磷 烯纳米片 (图 4) ${ }^{[16]}$ 。在以 Pt 纳米颗粒为助催化剂的 条件下, 这种磷烯纳米片可以从纯水 $(\mathrm{pH}=6.8)$ 中制 取氢气。此外, 采用温和的水热方法, 以红磷微球为 原料, 制备了具有层状多晶结构的磷烯纳米片 ${ }^{[17]}$ 。 将少量 Pt 纳米颗粒负载的磷烯分散在水和牺牲剂 (甲醇)的溶液中, 在可见光 $(\lambda>420 \mathrm{~nm})$ 下可得到较 高的光催化产氢活性 $\left(6.9 \mu \mathrm{mol} \cdot \mathrm{h}^{-1}\right)$, 是红磷的 203.2 倍 $\left(0.034 \mu \mathrm{mol} \cdot \mathrm{h}^{-1}\right)$, 且接近于 $3 \mathrm{wt} \% \mathrm{Pt}$ 沉积氮化碳 


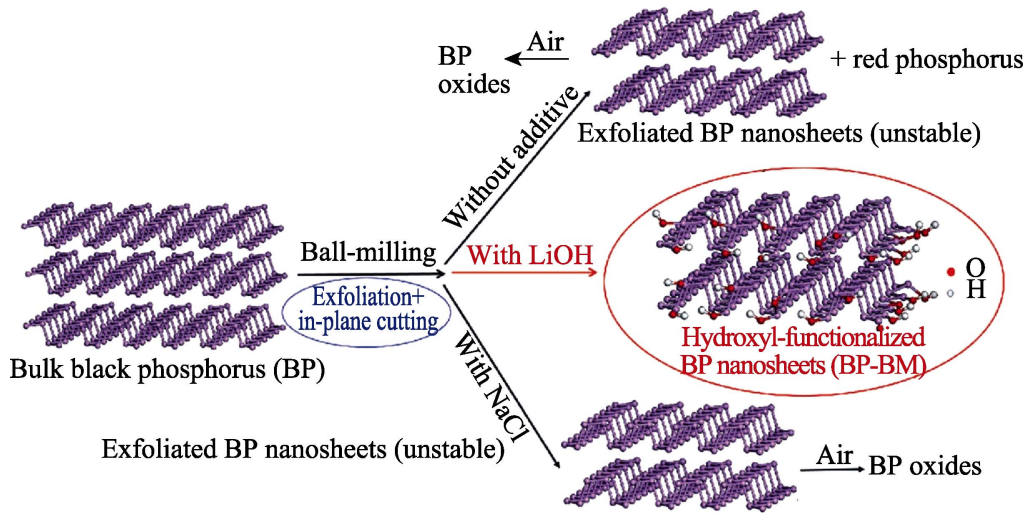

图 $3 \mathrm{BP}-\mathrm{BM}$ 纳米片的合成示意图 ${ }^{[15]}$

Fig. 3 Schematic diagram of the synthetic process of BP-BM nanosheets ${ }^{[15]}$

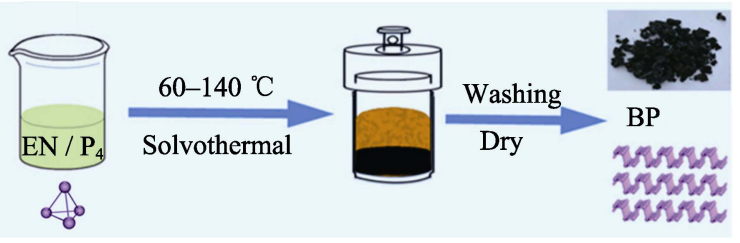

图 4 溶剂热法合成磷烯纳米片的示意图 ${ }^{[16]}$

Fig. 4 Schematic illustration of the preparation of few-layered BP nanosheets by a solvothermal process ${ }^{[16]}$

$\left(10 \mu \mathrm{mol} \cdot \mathrm{h}^{-1}\right)$ 。与红磷(带隙为 $\left.1.712 \mathrm{eV}\right)$ 相比, 磷烯纳 米片具有更高的光吸收能力(200 600 和 700 900 nm, 带隙为 $2.084 \mathrm{eV}$ ) 和光催化产氢活性。这些磷烯纳米 片具有光催化产氢的特性, 不仅为非金属单质光催 化剂引入了新的成员, 而且拓展了二维材料在太阳 能到氢能转化中的应用。

通过对磷烯进行表面修饰能够优化表面电子结 构，促进光生载流子的快速分离，改善表面反应动 力学, 提高磷烯的光催化分解水性能。将金属硫化 物、金属磷化物等产氢助催化剂负载于磷烯表面能 够实现光生电子和空穴的快速分离, 提高光催化产 氢效率。二硫化铇 $\left(\mathrm{WS}_{2}\right)$ 与 $\mathrm{BP}$ 的二维/二维 $(2 \mathrm{D} / 2 \mathrm{D})$ 复合光催化剂能在近红外 (NIR)光照射下实现光催 化分解水。 $\mathrm{BP} / \mathrm{WS}_{2}$ 复合物在 $>780 \mathrm{~nm}$ 和 $>808 \mathrm{~nm}$ 近 红外光照射下的产氢速率也明显高于 $\mathrm{BP}$ 和 $\mathrm{WS}_{2}{ }^{[18]}$ 。 在 $\mathrm{BP} / \mathrm{WS}_{2}$ 复合物中, 由于 $\mathrm{WS}_{2}$ 的功函数较低 $(5.57 \mathrm{eV}), \mathrm{BP}$ 中导带 $(\mathrm{CB})$ 上的电子被有效地注入到 $\mathrm{WS}_{2}$ 纳米薄片中。当足够多的电子被 $\mathrm{WS}_{2}$ 捕获时, 水被还原产生 $\mathrm{H}_{2}$ 。因此, $\mathrm{WS}_{2}$ 不仅可作为产氢助催 化剂, 而且加速了光生载流子的分离, 从而提高了分 解水的光催化活性 ${ }^{[18-19]}$ 。采用无定形磷化钴 (Co-P) 负载的 BP 纳米片作为光催化剂, 能够从纯水中制 取氢气 ${ }^{[20]}$ 。Co-P/BP 在纯水中表现出很高的氢气生 成率，在没有空穴牺牲剂和偏压的条件下，其在 $430 \mathrm{~nm}$ 处的产氢量子效率(AQE) 达到 $42.55 \%$, 在
$353 \mathrm{~K}$ 下的太阳能-氢能转换效率 $(\mathrm{STH})$ 超过 $5.4 \%$ 。 光催化活性的提高主要得益于 BP 纳米片对太阳能 的高效利用(约占太阳能的 $75 \%$ ) 以及 Co-P 对光生 载流子分离的促进作用 ${ }^{[21]}$ 。

此外, 采用氯化铟(III)、三(五氟苯基)硼烷和芐 基对 $\mathrm{BP}$ 进行表面功能化处理 ${ }^{[22]}$ 。功能化处理后的 BP 产氢速率最高可达 $6597 \mu \mathrm{mol} \cdot \mathrm{h}^{-1} \cdot \mathrm{g}^{-1}$, AQE 最高 可达 $8.4 \%$, 远高于未经功能化的 $\mathrm{BP}$ 。功能化处理 后的 BP 在水中拥有良好的分散性和稳定性。这些 表面修饰方法为制备高效、不含金属的产氢光催化 剂提供了新的策略。

\section{3 磷烯基异质结光催化剂的设计}

将半导体与 BP 复合形成异质结构可以增强光 吸收并提升光生载流子的分离效率，从而提高光催 化活性 ${ }^{[23-25]}$ 。

氮化碳聚合物(简称 $\mathrm{g}-\mathrm{C}_{3} \mathrm{~N}_{4}$ 或 $\mathrm{CN}$ )光催化剂已 在光解水制氢中得到广泛的研究 ${ }^{[26]}$ 。然而, 体相 $\mathrm{CN}$ 的光生电子-空穴对复合率高, 光催化制氢效率较 低 ${ }^{[27-28]}$ 。Zhu 等 ${ }^{[29]}$ 制备了由 $\mathrm{BP}$ 和 $\mathrm{CN}$ 纳米片组成 的纳米复合材料 $(\mathrm{BP} / \mathrm{CN})$, 将其作为非金属光催化 剂用于光催化制氢(图 5)。当以甲醇 $(\mathrm{MeOH})$ 为牺牲

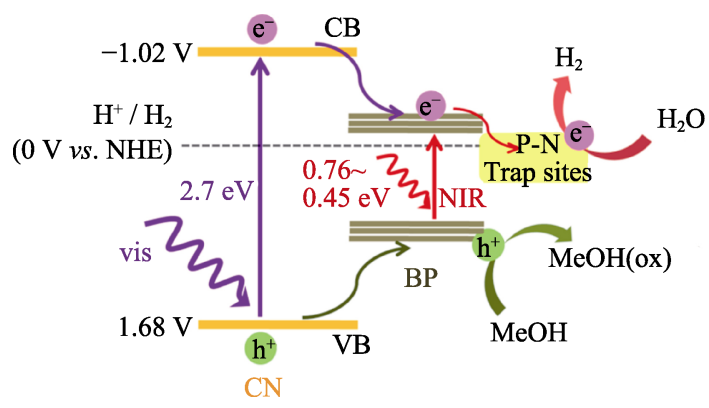

图 $5 \mathrm{BP} / \mathrm{CN}$ 催化剂在可见光和近红外光驱动下光催化分解 水的机理示意图 ${ }^{[29]}$

Fig. 5 Schematic diagram for the visible and NIR light driven photocatalytic $\mathrm{H}_{2}$ evolution reaction over $\mathrm{BP} / \mathrm{CN}$ catalyst ${ }^{[29]}$ 
剂时, 在 $\lambda>420 \mathrm{~nm}$ 和 $\lambda>780 \mathrm{~nm}$ 光照下均可观察到 $\mathrm{BP} / \mathrm{CN}$ 能够稳定地产生氢气。光催化制氢性能的提 升归因于 BP 和 CN 之间形成的强烈的界面相互作 用以及有效的界面电荷转移, 从而抑制光生载流子 的复合。虽然 $\mathrm{CN}$ 与 $\mathrm{BP}$ 纳米片之间形成的 I 型异 质结能延长光生载流子的寿命, 但是其光生电子和 空穴都集中在 $\mathrm{BP}$ 半导体上, 光生电子-空穴对的复 合几率仍然很高 ${ }^{[30-31]}$ 。

II 型异质结中的光生电子和空穴向相反方向迁 移并在不同的地方富集, 形成了光生载流子的空间 隔离, 从而有效抑制光生载流子的复合, 使更多的 光生电子能够参与到产氢反应中 ${ }^{[32]}$ 。Lei 等 ${ }^{[33]}$ 通过 超声处理方法将 $\mathrm{BP}$ 量子点 (BP QDs)负载于氮化碳 $(\mathrm{CN})$ 纳米片上以构筑 II 型异质结。所合成的 $\mathrm{BP}$ $\mathrm{QDs} / \mathrm{CN}$ 复合材料的可见光驱动制氢性能随 BP QDs 含量的变化而变化。5 $\mathrm{wt} \% \mathrm{BP} \mathrm{QDs} / \mathrm{CN}$ 具有最优的 析氢速率, 达到 $271 \mu \mathrm{mol} \cdot \mathrm{h}^{-1} \cdot \mathrm{g}^{-1}$, 分别比原始 $\mathrm{CN}$ 和 BP QDs 高 5.6 和 4.2 倍。II 型异质结、P-C 键的形 成以及 $\mathrm{BP} \mathrm{QDs}$ 和 $\mathrm{CN}$ 之间有效的界面电荷分离与 传递协同增强了光催化活性和光稳定性。

此外, 磷烯也可作为一种非金属的助催化剂用 于提高金属硫化物在光催化分解水中的活性。乔 世璋课题组 ${ }^{[34]}$ 制备了少层磷烯纳米片与金属硫化 物 $\left(\mathrm{CdS}, \mathrm{Zn}_{0.8} \mathrm{Cd}_{0.2} \mathrm{~S}\right.$ 和 $\mathrm{ZnS}$ )的复合光催化剂(图 6)。 当将 $1.0 \mathrm{wt} \%$ 少层磷烯纳米片和 $\mathrm{CdS}$ 通过简单的机 械混合后, 材料在可见光光催化产氢效率方面有 了极大的改善, 在 $>420 \mathrm{~nm}$ 可见光下的产氢活性达到 了 $11192 \mu \mathrm{mol} \cdot \mathrm{h}^{-1} \cdot \mathrm{g}^{-1}$, 在 $420 \mathrm{~nm}$ 处的 $\mathrm{AQE}$ 为 $34.7 \%$ 。 出色的活性来自于磷烯和 $\mathrm{CdS}$ 之间形成的 II 型异质 结、强的电子耦合、合适的能带结构、高载流子迁 移率和磷烯表面大量的活性位点。虽然 II 型异质结 构能有效促进光生电子-空穴的分离, 但是其降低 了光生电子的还原能力和空穴的氧化能力。

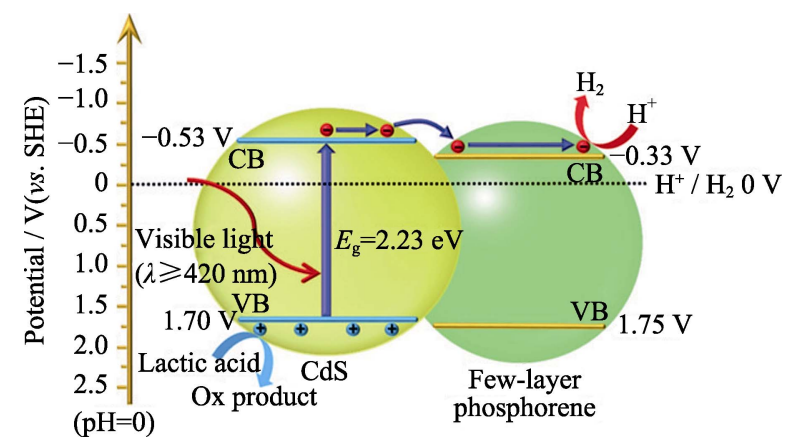

图 $6 \mathrm{CdS}$ 与 $\mathrm{BP}$ 复合催化剂的光催化分解水的机理示意图 ${ }^{[34]}$ Fig. 6 Schematic illustration of the mechanism of CdS and BP hybrid catalyst for photocatalytic water splitting ${ }^{[34]}$
$\mathrm{Z}$ 型异质结通过控制界面的载流子迁移使低能 量的光生电子与空穴直接复合, 保留高能量的光生 电子-空穴, 从而大大提高了光催化效率。 $\mathrm{Hu}$ 等 ${ }^{[35]}$ 制备了 $\mathrm{BP} /$ 单层 $\mathrm{Bi}_{2} \mathrm{WO}_{6}$ 的 $2 \mathrm{D} / 2 \mathrm{D} \mathrm{Z}$ 型异质结。这 种 $\mathrm{BP} / \mathrm{Bi}_{2} \mathrm{WO}_{6}$ 异质结在光催化分解水制氢方面表现 出较好的光催化性能。 $\mathrm{BP} / \mathrm{Bi}_{2} \mathrm{WO}_{6}$ 的最高产氢速率 为 $21042 \mu \mathrm{mol} \cdot \mathrm{g}^{-1}$, 是 $\mathrm{Bi}_{2} \mathrm{WO}_{6}$ 的 9.2 倍。 $\mathrm{BP} / \mathrm{Bi}_{2} \mathrm{WO}_{6}$ 异质结具有良好的光催化性能, 主要归功于 $2 \mathrm{D} / 2 \mathrm{D}$ 异质结的界面协同效应、高效的电荷分离和转移以 及强的吸光性 ${ }^{[35]}$ 。此外, 通过上转换材料与 BP 复 合的方式可增强催化剂对近红外光的利用。Zhang 等 ${ }^{[36]}$ 合成了 $\mathrm{BP}$ 修饰的三维 $\beta-\mathrm{NaYF}_{4}: \mathrm{Yb}^{3+}$, $\mathrm{Tm}^{3+} @ \mathrm{Ag}_{3} \mathrm{PO}_{4}$ 微棒 $\left(\mathrm{NYF} @ \mathrm{Ag}_{3} \mathrm{PO}_{4} @ \mathrm{BP}\right)$, 并将其作 为一种高效的近红外光响应的 $\mathrm{Z}$ 型光催化剂。在 $980 \mathrm{~nm}$ 的激光照射下, $\mathrm{NaYF}_{4}: \mathrm{Yb}^{3+}, \mathrm{Tm}^{3+}$ 上转换材料 能将 $980 \mathrm{~nm}$ 的激光转化为可见光和紫外光, 并被 $\mathrm{Ag}_{3} \mathrm{PO}_{4}$ 和 $\mathrm{BP}$ 利用。NYF@ $@ \mathrm{Ag}_{3} \mathrm{PO}_{4} @ \mathrm{BP}$ 复合材料在 析氢过程中表现出更高的光催化活性, 其产氢速率 比 BP 高 10 倍，比 NYF@BP 高 6 倍。这种复合材料 的 $\mathrm{Z}$ 型电子迁移机制提升了光生载流子的分离效率, 增强了氧化还原能力, 从而提高了光催化活性 ${ }^{[36]}$ 。

石墨烯是一种具有高比表面积和载流子迁移率 的二维材料。石墨烯/BP 复合材料可作为一种高效 的分解水光催化剂 ${ }^{[37]}$ 。在 $\mathrm{Pt}$ 纳米粒子和还原石墨烯 氧化物 $(\mathrm{RGO}) 、 \mathrm{BP}$ 纳米片存在的条件下, $\mathrm{BP} / \mathrm{Pt} / \mathrm{RGO}$ 体系在 $>420 \mathrm{~nm}$ 的可见光和 $>780 \mathrm{~nm}$ 的近红外光下 光照 $4 \mathrm{~h}$ 后分别得到约 5.13 和 $1.26 \mu \mathrm{mol}$ 的 $\mathrm{H}_{2}$, 而 且在 $(420 \pm 5)$ 和 $(780 \pm 5) \mathrm{nm}$ 处的 AQE 分别为 $8.7 \%$ 和 $1.5 \%$ 。 Pt $/ \mathrm{RGO}$ 与 $\mathrm{BP}$ 纳米粒子之间存在有效的电荷 分离和迁移, 有助于提升可见光和近红外光下的产 氢效率 ${ }^{[37]}$ 。此外, 二硫化钼 $\left(\mathrm{MoS}_{2}\right)-\mathrm{BP} /$ 氧化石墨烯 (GO) 的三元光催化剂在光催化分解水制氢反应中 也具有良好的应用前景 ${ }^{[38]}$, 在可见-近红外光照射 下的光催化产氢活性达到了 $3.47 \mu \mathrm{mol} \cdot \mathrm{h}^{-1}$, 比 $\mathrm{BP} / \mathrm{GO}$ 和 $\mathrm{MoS}_{2}$ - $\mathrm{BP}$ 分别提高了 13.3 和 2.27 倍。GO 在此具 有双重功能: 一方面, GO 在合成过程中充当表面活 性剂和催化剂载体, 有利于促进 $\mathrm{BP}$ 的剥离以及 $\mathrm{MoS}_{2}$ 助催化剂在 $\mathrm{BP} / \mathrm{GO}$ 表面上的修饰; 另一方面, 由于其局部共轭芳族体系和良好的电子穿梭能力, GO 在光催化反应过程中有效地促进了电荷的分离 和转移, 从而提高了光催化产氢性能 ${ }^{[38]}$ 。

等离子共振光催化剂在光催化分解水中也具有 重要应用。利用 $\mathrm{Au}$ 纳米颗粒的等离子体共振(SPR) 和 2D-BP 较宽广的带隙范围(0.3 2.1 eV), 可对 $\mathrm{La}_{2} \mathrm{Ti}_{2} \mathrm{O}_{7}(\mathrm{LTO})$ 进行敏化 ${ }^{[39]}$ 。在可见光 $(>420 \mathrm{~nm})$ 和近 红外光 $(>780 \mathrm{~nm})$ 的激发下, $\mathrm{BP}-\mathrm{Au} / \mathrm{La}_{2} \mathrm{Ti}_{2} \mathrm{O}_{7}$ 的产氢 速率可达 0.74 和 $0.30 \mathrm{mmol} \cdot \mathrm{h}^{-1} \cdot \mathrm{g}^{-1}$ 。如图 7 所示, $\mathrm{Au}$ 


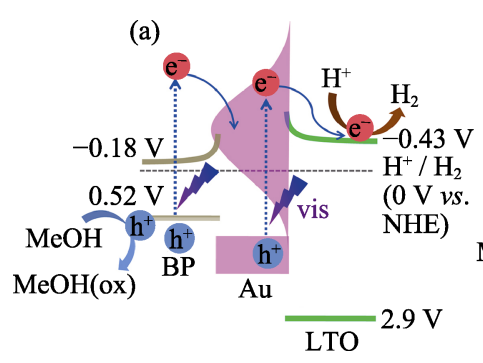

(b)

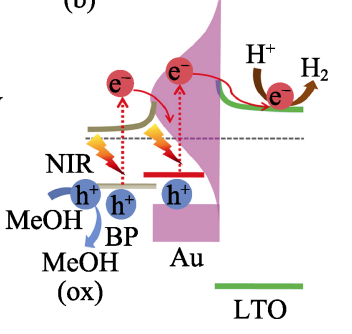

下可观察到纯水分解产生氢气和氧气。 $\mathrm{BP} / \mathrm{BiVO}_{4}$ 能带结构的交错排列有助于电荷分离, 使得水的还 原和氧化分别发生于 $\mathrm{BP}$ 和 $\mathrm{BiVO}_{4}$ 。这些结果预示 了 $\mathrm{BP}$ 基复合材料在全水分解反应领域的应用前景。

\section{3 磷烯在其他光催化领域中的应用}

图 $7 \mathrm{BP}-\mathrm{Au} / \mathrm{LTO}$ 在(a)可见光和(b)近红外光照射下光催化制 取氢气的原理图 ${ }^{[39]}$

Fig. 7 Schematic diagrams of photocatalytic $\mathrm{H}_{2}$ production using BP-Au/LTO under (a)visible and (b)NIR light irradiation ${ }^{[39]}$

和 BP 受可见光和近红外光激发后将电子转移注入 LTO, 而 LTO 可以在甲醇牺牲剂存在下高效催化产 生 $\mathrm{H}_{2}$ 。 $\mathrm{BP}$ 和等离子体 $\mathrm{Au}$ 的宽光谱吸收以及激发的 $\mathrm{BP}$ 和 $\mathrm{Au}$ 向 $\mathrm{La}_{2} \mathrm{Ti}_{2} \mathrm{O}_{7}$ 的有效界面电子转移使其在可 见光区和近红外光区的光催化活性显著提高。

这些 I 型、II 型、 $Z$ 型异质结光催化剂的设计 将为高效太阳能制氢体系的构建提供一条新的思路, 而上转换、等离子体共振效应的耦合作用也将对发 展全太阳光谱响应的高活性光催化剂具有重要的参 考价值 ${ }^{[40-43]}$ 。

\section{2 磷烯光催化分解水产氧和全水分解 反应}

除产氢半反应外, BP 基材料还被应用于水的氧 化半反应。Yan 等 ${ }^{[44]}$ 利用 BP-Ni(OH $)_{2}$ 作为水氧化的 有效光催化剂, 在模拟太阳光照射下、 $0.1 \mathrm{~mol} \cdot \mathrm{L}^{-1}$ 的 $\mathrm{Na}_{2} \mathrm{~S}_{2} \mathrm{O}_{4}$ 溶液中, 产氧速率可达 $15.7 \mu \mathrm{mol} \cdot \mathrm{g}^{-1}$ catalyst $\mathrm{h}^{-1}$ ) 或 $224.3 \mu \mathrm{mol} \cdot \mathrm{g}^{-1} \mathrm{BP}^{\circ} \cdot \mathrm{h}^{-1}$ )。光生空穴与电子分别迁移 到 $\mathrm{Ni}(\mathrm{OH})_{2}$ 与 $\mathrm{BP}$ 的表面, 并促进光催化水氧化反应 的进行。此外, $\mathrm{Zhu}$ 等 ${ }^{[45]}$ 构筑了 $\mathrm{BP}$ 和 $\mathrm{BiVO}_{4}$ 的 $2 \mathrm{D} /$ $2 \mathrm{D}$ 异质结构, 并构建了高效 $\mathrm{Z}$ 型光催化全水分解体 系(图 8)。在没有任何牺牲试剂和外加偏压的情况下, 使用 $\mathrm{BP} / \mathrm{BiVO}_{4}$ 异质结构光催化剂, 在可见光照射

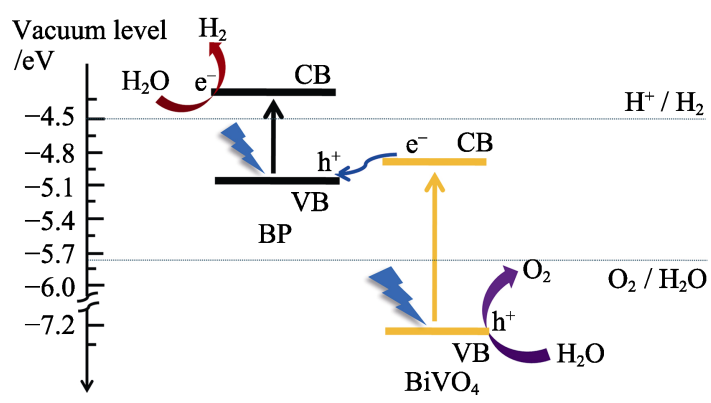

图 8 可见光照射下 $\mathrm{BP} / \mathrm{BiVO}_{4}$ 的 $\mathrm{Z}$ 型光催化裂解水系统原 理图 ${ }^{[45]}$

Fig. 8 Schematic diagram of Z-scheme photocatalytic water splitting system using $\mathrm{BP} / \mathrm{BiVO}_{4}$ under visible light irradiation ${ }^{[45]}$
除了光催化分解水以外, 磷烯在光催化活化分 子氧、污染物降解、 $\mathrm{CO}_{2}$ 还原、 $\mathrm{H}_{2} \mathrm{O}_{2}$ 合成、合成氨、 有机选择性合成等方面也有潜在的应用前景。本课 题组 ${ }^{[46]}$ 通过超声辅助一步液相剥层法成功制备了 磷烯和聚合物氮化碳组成的 $\mathrm{BP} / \mathrm{CN}$ 复合光催化剂。 $\mathrm{BP}$ 与 $\mathrm{CN}$ 复合增强了可见光吸收能力, 促进了光催 化过程中电荷分离, 提升了光诱导的分子氧活化能 力, 加速了超氧自由基 $\left(\cdot \mathrm{O}_{2}{ }^{-}\right.$)和 $\mathrm{H}_{2} \mathrm{O}_{2}$ 的产生(图 9)。 当 $\mathrm{BP}$ 含量为 $10 \mathrm{wt} \%$ 时, $\mathrm{BP} / \mathrm{CN}$ 复合光催化剂降解罗 丹明 $\mathrm{B}$ 的活性最佳, 在可见光下照射 $15 \mathrm{~min}$ 可分解 $98 \%$ 的罗丹明 B 染料, 其降解的速率常数约是单独 $\mathrm{CN}$ 的 4 倍。该工作强调了将能级匹配的半导体相 复合可显著提升光催化剂产生活性氧物种的性能, 揭示了构建异质结以提升光催化活性的广阔前景, 在环境治理和能源转化领域具有巨大的应用潜力。
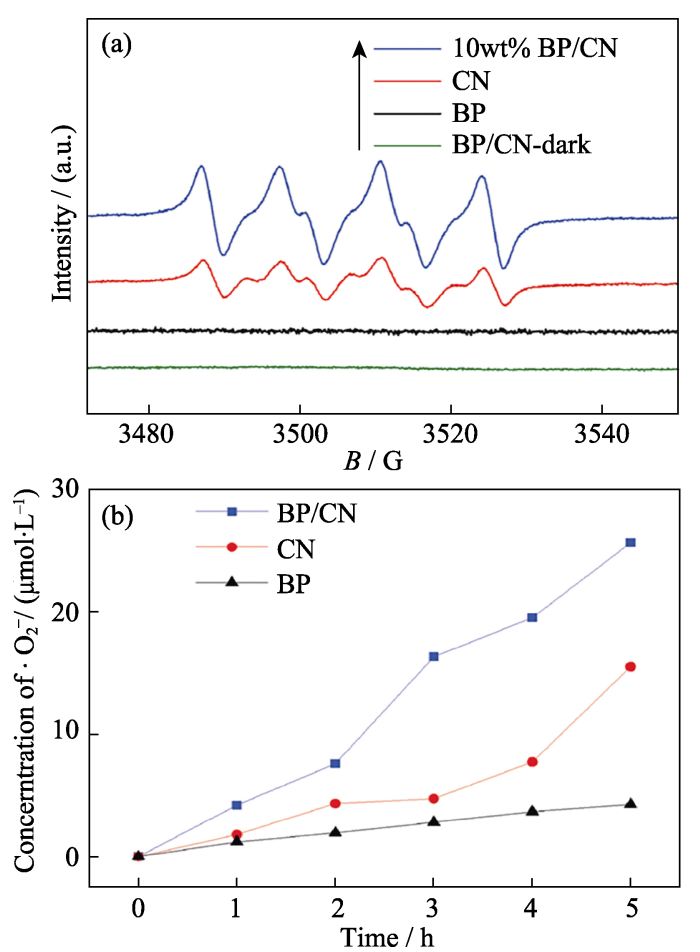

图 9 (a)BP/CN 异质结构光催化剂在可见光照射下产生超氧 自由基 $\left(\cdot \mathrm{O}_{2}{ }^{-}\right)$的电子自旋共振谱图, (b)在可见光照射下降解四 唑氮蓝溶液以测定 $\mathrm{BP} / \mathrm{CN}$ 复合材料产生 $\cdot \mathrm{O}_{2}{ }^{-}$的性能曲线 ${ }^{[46]}$

Fig. 9 (a) Electron spin resonance spectra of $\cdot \mathrm{O}_{2}{ }^{-}$radicals over $\mathrm{BP} / \mathrm{CN}$ hybrid with visible-light irradiation, and (b) timedependent degradation of nitroblue tetrazolium solution to detect $\cdot \mathrm{O}_{2}{ }^{-}$evolution over $\mathrm{BP} / \mathrm{CN}$ hybrid under visible-light irradiation $^{[46]}$ 


\section{4 结论与展望}

本文总结了磷烯及其异质结构在光催化分解水 产氢、产氧和全水分解方面的研究进展, 重点关注 催化剂的设计原则、改性方法、构-效关系和电荷转 移机制, 揭示了磷烯材料在太阳能利用和转化方面 的重要应用潜力。尽管磷烯基异质结构光催化剂取 得了显著的进展, 但其光催化效率仍相对较低, 机 理不够明确, 今后仍需要在以下几个方面继续开展 研究工作: 首先, 探索合适的方法来提高磷烯的光 催化性能。对磷烯的能带结构和表面性质进行调控, 并通过合理的制备方法获得更好的界面接触效果， 从而提高光催化分解水性能。其次, 拓展磷烯在多 种光催化反应中的应用。虽然磷烯在光催化分解水 产氢和污染物降解方面取得了巨大的进展, 但在全 水分解、 $\mathrm{CO}_{2}$ 还原和有机合成方面的应用研究还远 远不够。通过构建异质结并与其他助催化剂偶联, 构筑高效的光催化体系, 拓展磷烯在多种光催化反 应中的应用, 将为人工光合成开辟新的途径 ${ }^{[47-48]}$ 。 再次，深入了解构效关系和光催化反应机理。通过 模拟计算和原位表征方法, 在分子水平上理解光催 化反应的机理以及催化剂结构与性能之间的关 系 ${ }^{[49-50]}$, 从而对新型磷烯基光催化剂的设计和制备 提供理论指导, 促进磷烯材料的发展。最后, 增强磷 烯材料在光催化反应中的稳定性。磷烯的氧化是由 $\mathrm{O}_{2}$ 与磷烯表面的孤对电子发生反应引起的，导致磷 氧化物的生成。寻找合适有效的方法来增强磷烯在 光催化反应中的长期稳定性仍是一项巨大的挑 战 ${ }^{[51-52]}$ 。磷烯基异质结材料在光(电)催化领域将具 有广阔的应用前景, 有望促进二维纳米材料在能源 转换中的开发和应用 ${ }^{[53-55]}$ 。

\section{参考文献:}

[1] FUJISHIMA A, HONDA K. Electrochemical photolysis of water at a semiconductor electrode. Nature, 1972, 238(5358): 37-38.

[2] ZHENG D, ZHANG G, WANG X. Integrating CdS quantum dots on hollow graphitic carbon nitride nanospheres for hydrogen evolution photocatalysis. Appl. Catal. B-Environ, 2015, 179: 479-488.

[3] LU Q, HUA L, CHEN Y, et al. Preparation and property of oxygendeficient $\mathrm{Bi}_{2} \mathrm{WO}_{6-x}$ photocatalyst active in visible light. J. Inorg. Mater, 2015, 30(4): 413-419.

[4] WAN J, HU D, LU P, et al. Preparation of anatase $\mathrm{TiO}_{2}$ nanocube with exposed (001) facet and its photocatalytic properties. J. Inorg. Mater., 2016, 31(8): 845-849.

[5] YI Z, YE J, KIKUGAWA N, et al. An orthophosphate semiconductor with photooxidation properties under visible-light irradiation. Nat. Mater., 2010, 9: 559.

[6] WANG X, MAEDA K, THOMAS A, et al. A metal-free polymeric photocatalyst for hydrogen production from water under visible light. Nat. Mater, 2008, 8: 76.

[7] BAI Y, WILBRAHAM L, SLATER B J, et al. Accelerated discovery of organic polymer photocatalysts for hydrogen evolution from water through the integration of experiment and theory. $J . A m$. Chem. Soc., 2019, 141(22): 9063-9071.

[8] ZHAO C, CHEN Z, XU J, et al. Probing supramolecular assembly and charge carrier dynamics toward enhanced photocatalytic hydrogen evolution in 2D graphitic carbon nitride nanosheets. Appl. Catal. B-Environ, 2019, 256: 117867.

[9] YANG X, TIAN L, ZHAO X, et al. Interfacial optimization of g$\mathrm{C}_{3} \mathrm{~N}_{4}$-based Z-scheme heterojunction toward synergistic enhancement of solar-driven photocatalytic oxygen evolution. Appl. Catal. B-Environ, 2019, 244: 240-249.

[10] LI J, LI H, ZHAN G, et al. Solar water splitting and nitrogen fixation with layered bismuth oxyhalides. Acc. Chem. Res., 2017, 50(1): $112-121$.

[11] LI B, LAI C, ZENG G, et al. Black phosphorus, a rising star 2D nanomaterial in the post-graphene era: synthesis, properties, modifications, and photocatalysis applications. Small, 2019, 15(8): 1804565.

[12] YAN J, VERMA P, KUWAHARA Y, et al. Recent progress on black phosphorus-based materials for photocatalytic water splitting. Small Methods, 2018, 2(12): 1800212.

[13] SA B, LI Y L, QI J, et al. Strain engineering for phosphorene: the potential application as a photocatalyst. J. Phys. Chem. C, 2014, 118(46): 26560-26568.

[14] RAHMAN MZ, BATMUNKH M, BAT-ERDENE M, et al. p-type $\mathrm{BP}$ nanosheet photocatalyst with $\mathrm{AQE}$ of $3.9 \%$ in the absence of a noble metal cocatalyst: investigation and elucidation of photophysical properties. J. Mater. Chem. A, 2018, 6(38): 18403-18408.

[15] ZHU X, ZHANG T, SUN Z, et al. Black phosphorus revisited: a missing metal-free elemental photocatalyst for visible light hydrogen evolution. Adv. Mater., 2017, 29(17): 1605776.

[16] TIAN B, TIAN B, SMITH B, et al. Facile bottom-up synthesis of partially oxidized black phosphorus nanosheets as metal-free photocatalyst for hydrogen evolution. Proc. Natl. Acad. Sci., 2018, 115(17): 4345-4350.

[17] ZHAO G, WANG T, SHAO Y, et al. A novel mild phase-transition to prepare black phosphorus nanosheets with excellent energy applications. Small, 2017, 13(7): 1602243.

[18] ZHU M, ZHAI C, FUJITSUKA M, et al. Noble metal-free nearinfrared-driven photocatalyst for hydrogen production based on 2D hybrid of black phosphorus/WS $\mathrm{WS}_{2}$. Appl. Catal. B-Environ, 2018, 221: 645-651.

[19] YUAN Y J, WANG P, LI Z, et al. The role of bandgap and interface in enhancing photocatalytic $\mathrm{H}_{2}$ generation activity of 2D-2D black phosphorus $/ \mathrm{MoS}_{2}$ photocatalyst. Appl. Catal. B-Environ, 2019, 242: 1-8.

[20] TIAN B, TIAN B, SMITH B, et al. Supported black phosphorus nanosheets as hydrogen-evolving photocatalyst achieving 5.4\% energy conversion efficiency at 353 K. Nat. Commun., 2018, 9: 1397.

[21] LIANG Q, SHI F, XIAO X, et al. In situ growth of CoP nanoparticles anchored on black phosphorus nanosheets for enhanced photocatalytic hydrogen production. ChemCatChem, 2018, 10(10): 2179-2183.

[22] VISHNOI P, GUPTA U, PANDEY R, et al. Stable functionalized phosphorenes with photocatalytic HER activity. J. Mater. Chem. A, 2019, 7(12): 6631-6637.

[23] WU J, HUANG S, JIN Z, et al. Black phosphorus: an efficient cocatalyst for charge separation and enhanced photocatalytic hydrogen evolution. J. Mater. Sci., 2018, 53(24): 16557-16566. 
[24] ELBANNA O, ZHU M, FUJITSUKA M, et al. Black phosphorus sensitized $\mathrm{TiO}_{2}$ mesocrystal photocatalyst for hydrogen evolution with visible and near-infrared light irradiation. ACS Catal., 2019, 9(4): 3618-3626

[25] ZHAO H, LIU H, SUN R, et al. A $\mathrm{Zn}_{0.5} \mathrm{Cd}_{0.5} \mathrm{~S}$ photocatalyst modified by $2 \mathrm{D}$ black phosphorus for efficient hydrogen evolution from water. ChemCatChem, 2018, 10(19): 4395-4405.

[26] ZHENG Y, LIN L, YE X, et al. Helical graphitic carbon nitrides with photocatalytic and optical activities. Angew. Chem. Int. Ed., 2014, 53(44): 11926-11930.

[27] ZHENG Y, LIN L, WANG B, et al. Graphitic carbon nitride polymers toward sustainable photoredox catalysis. Angew. Chem. Int. $E d ., 2015, \mathbf{5 4}(44):$ 12868-12884.

[28] ZHENG Y, YU Z, LIN F, et al. Sulfur-doped carbon nitride polymers for photocatalytic degradation of organic pollutant and reduction of $\mathrm{Cr}(\mathrm{VI})$. Molecules, 2017, 22(4): 572.

[29] ZHU M, KIM S, MAO L, et al. Metal-free photocatalyst for $\mathrm{H}_{2}$ evolution in visible to near-infrared region: black phosphorus/graphitic carbon nitride. J. Am. Chem. Soc., 2017, 139(37): 13234- 13242.

[30] WEN M, WANG J, TONG R, et al. A low-cost metal-free photocatalyst based on black phosphorus. Adv. Sci., 2019, 6(1): 1801321

[31] RAN J R, GUO W W, WANG H L, et al. Metal-free 2D/2D phosphorene/g- $\mathrm{C}_{3} \mathrm{~N}_{4}$ van der Waals heterojunction for highly enhanced visible-light photocatalytic $\mathrm{H}_{2}$ production. Adv. Mater, 2018, 30(25): 1800128

[32] DU H, LIU Y, SHEN C, et al. Nanoheterostructured photocatalysts for improving photocatalytic hydrogen production. Chin. J. Catal., 2017, 38(8): 1295-1306

[33] LEI W, MI Y, FENG R, et al. Hybrid 0D-2D black phosphorus quantum dots-graphitic carbon nitride nanosheets for efficient hydrogen evolution. Nano Energy, 2018, 50: 552-561.

[34] RAN J, ZHU B, QIAO S Z. Phosphorene co-catalyst advancing highly efficient visible-light photocatalytic hydrogen production. Angew. Chem. Int. Ed., 2017, 56(35): 10373-10377.

[35] HU J, CHEN D, MO Z, et al. Z-scheme 2D/2D heterojunction of black phosphorus/monolayer $\mathrm{Bi}_{2} \mathrm{WO}_{6}$ nanosheets with enhanced photocatalytic activities. Angew. Chem. Int. Ed., 2019, 58(7): 2073-2077.

[36] ZHANG Y, WANG L, PARK S H, et al. Single near-infrared-laser driven Z-scheme photocatalytic $\mathrm{H}_{2}$ evolution on upconversion material@ $\mathrm{Ag}_{3} \mathrm{PO}_{4} @$ black phosphorus. Chem. Eng. J., 2019, 375: 121967.

[37] ZHU M, OSAKADA Y, KIM S, et al. Black phosphorus: a promising two dimensional visible and near-infrared-activated photocatalyst for hydrogen evolution. Appl. Catal. B-Environ, 2017, 217: 285-292.

[38] ZHU M, FUJITSUKA M, ZENG L, et al. Dual function of graphene oxide for assisted exfoliation of black phosphorus and electron shuttle in promoting visible and near-infrared photocatalytic $\mathrm{H}_{2}$ evolution. Appl. Catal. B-Environ, 2019, 256: 117864 .

[39] ZHU M, CAI X, FUJITSUKA M, et al. $\mathrm{Au} / \mathrm{La}_{2} \mathrm{Ti}_{2} \mathrm{O}_{7}$ nanostructures sensitized with black phosphorus for plasmon-enhanced photocatalytic hydrogen production in visible and near-infrared light. Angew. Chem. Int. Ed., 2017, 56(8): 2064-2068.

[40] RAN J, WANG X, ZHU B, et al. Strongly interactive 0D/2D het- erostructure of a $\mathrm{Zn}_{x} \mathrm{Cd}_{1-x} \mathrm{~S}$ nanoparticle decorated phosphorene nanosheet for enhanced visible-light photocatalytic $\mathrm{H}_{2}$ production. Chem. Commun., 2017, 53(71): 9882-9885.

[41] REDDY DA, KIM E H, GOPANNAGARI M, et al. Few layered black phosphorus $/ \mathrm{MoS}_{2}$ nanohybrid: a promising co-catalyst for solar driven hydrogen evolution. Appl. Catal. B-Environ, 2019, 241: 491-498.

[42] BOPPELLA R, YANG W, TAN J, et al. Black phosphorus supported $\mathrm{Ni}_{2} \mathrm{P}$ co-catalyst on graphitic carbon nitride enabling simultaneous boosting charge separation and surface reaction. Appl. Catal. B-Environ, 2019, 242: 422-430.

[43] $\mathrm{HU}$ J, JI Y, MO Z, et al. Engineering black phosphorus to porous $\mathrm{g}_{-} \mathrm{C}_{3} \mathrm{~N}_{4}$-metal-organic framework membrane: a platform for highly boosting photocatalytic performance. J. Mater. Chem. A, 2019, 7(9): 4408-4414.

[44] YAN J, JI Y, KONG L, et al. Black phosphorus-based compound with few layers for photocatalytic water oxidation. Chem CatChem, 2018, 10(16): 3424-3428.

[45] ZHU M, SUN Z, FUJITSUKA M, et al. Z-scheme photocatalytic water splitting on a $2 \mathrm{D}$ heterostructure of black phosphorus/bismuth vanadate using visible light. Angew. Chem. Int. Ed., 2018, 57(8): 2160-2164.

[46] ZHENG Y, YU Z, OU H, et al. Black phosphorus and polymeric carbon nitride heterostructure for photoinduced molecular oxygen activation. Adv. Funct. Mater., 2018, 28(10): 1705407.

[47] RUDENKO A N, KATSNELSON M I. Quasiparticle band structure and tight-binding model for single- and bilayer black phosphorus. Phys. Rev. B, 2014, 89(20): 201408.

[48] TRAN V, SOKLASKI R, LIANG Y, et al. Layer-controlled band gap and anisotropic excitons in few-layer black phosphorus. Phys. Rev. B, 2014, 89(23): 235319.

[49] ZHOU Q, CHEN Q, TONG Y, et al. Light-induced ambient degradation of few-layer black phosphorus: mechanism and protection. Angew. Chem. Int. Ed., 2016, 55(38): 11437-11441.

[50] QIU M, WANG D, LIANG W, et al. Novel concept of the smart NIR-light-controlled drug release of black phosphorus nanostructure for cancer therapy. Proc. Natl. Acad. Sci., 2018, 115(3): 501-506.

[51] PENG X, WEI Q, COPPLE A. Strain-engineered direct-indirect band gap transition and its mechanism in two-dimensional phosphorene. Phys. Rev. B, 2014, 90(8): 085402

[52] TANG X, LIANG W, ZHAO J, et al. Fluorinated phosphorene: electrochemical synthesis, atomistic fluorination, and enhanced stability. Small, 2017, 13(47): 1702739.

[53] MAO L, CAI X, YANG S, et al. Black phosphorus-CdS- $-\mathrm{La}_{2} \mathrm{Ti}_{2} \mathrm{O}_{7}$ ternary composite: effective noble metal-free photocatalyst for full solar spectrum activated $\mathrm{H}_{2}$ production. Appl. Catal. B-Environ, 2019, 242: 441-448.

[54] FENG R, LEI W, LIU G, et al. Visible- and NIR-light responsive black-phosphorus-based nanostructures in solar fuel production and environmental remediation. Adv. Mater, 2018, 30(49): 1804770

[55] ZHANG K, JIN B, PARK C, et al. Black phosphorene as a hole extraction layer boosting solar water splitting of oxygen evolution catalysts. Nat. Commun., 2019, 10(1): 2001 